\title{
Weight change and its correlates among breast cancer survivors
}

\author{
Xiaoli Chen ${ }^{1}$, Wei Lu², Kai Gu², Zhi Chen ${ }^{1}$, Ying Zheng ${ }^{2}$, Wei Zheng ${ }^{1}$, and Xiao Ou Shu ${ }^{1}$ \\ ${ }^{1}$ Department of Medicine, Vanderbilt Epidemiology Center, Vanderbilt University Medical Center \\ and Vanderbilt-Ingram Cancer Center, 2525 West End Avenue, Suite 600, Nashville, TN 37203, \\ USA
}

2 Shanghai Institute of Preventive Medicine, 1380 Zhong Shan Road (W), Shanghai, 200336, China

\begin{abstract}
We assessed weight change from diagnosis to approximately 18 months after cancer diagnosis and evaluated its correlates in a large, population-based, cohort study of women diagnosed with stage 0 -IV breast cancer. A total of 4561 cases with weight information 1 year prior to diagnosis, at diagnosis, and at the 18-month post-diagnosis interview were included in the study. Multinomial logistic regression models were conducted to examine the association of weight change from diagnosis to 18 months after diagnosis with sociodemographic, clinical, and lifestyle factors. The mean weight change from diagnosis to 18 months after diagnosis was a gain of $1.7 \mathrm{~kg}$ (median: 2.0). Overall, $61 \%$ of women gained weight, $27 \%$ gained $2-5 \mathrm{~kg}$, and $24 \%$ gained $\geq 5 \mathrm{~kg}$, while approximately $14 \%$ lost $>2 \mathrm{~kg}$ during the 18 -month post-diagnosis period. Greater weight gain was significantly related to younger age, premenopausal status, mixed receptor status, more advanced disease stage, pre-diagnosis weight loss, higher dietary intake, and cigarette smoking. Women with obesity and serious comorbidity were more likely to lose weight. Moderate exercise was not significantly related to weight change. Weight gain is common among breast cancer survivors. Sociodemographic, clinical, and lifestyle factors are related to weight change. Appropriate intervention strategies should be developed.
\end{abstract}

\section{Keywords}

weight change; breast cancer survivor; lifestyle; risk factor

\section{Introduction}

Weight gain is a profound issue among breast cancer patients and survivors [1-3] and usually ranges from 1.0 to $6.0 \mathrm{~kg}$ during the first year after a diagnosis of breast cancer $[1,2$, 4-7]. Weight gain after breast cancer diagnosis may have a negative impact on quality of life, increase the risk for recurrence, and shorten survival time [2, 3, 7-9]. Several factors have been suggested to influence weight gain after cancer diagnosis, including age $[6,10]$, development of menopause $[1,6,10]$, advanced disease stage [6], cancer-related treatments $[1,6,10]$, decreased physical activity $[2,6,10]$, and increased energy intake $[2,10]$. However, the evidence is not entirely consistent [1, 2, 6, 10-12].

Most previous studies on weight gain and related factors after breast cancer diagnosis have been cross-sectional, had a short study duration, or had small sample sizes $[1,5,6,11$,

Corresponding Author: Xiao Ou Shu, M.D., Ph.D., Professor of Medicine, Vanderbilt Epidemiology Center, 2525 West End Avenue, Suite 600, IMPH, Nashville, TN 37203-1738, Tel: 615-936-0713, Fax: 615-936-8291, xiaoou.shu@vanderbilt.edu. 
13-15]. Few studies have considered the association of demographic, clinical, and lifestyle factors with weight gain concurrently $[6,15]$, especially in large, population-based, cohort studies [6]. Weight loss before cancer diagnosis may have a major effect on weight change after cancer diagnosis, and may lead to a regaining weight after cancer-related treatments. However, few studies have examined the effect of pre-diagnosis weight loss on postdiagnosis weight change. Furthermore, most studies on weight change have been conducted in Western countries where the prevalence of obesity is high [1, 2, 4-6]. For example, in a study of 1169 breast cancer patients who received chemotherapy before surgery in the US, $32 \%$ of patients were overweight and $30 \%$ were obese [16]. It is possible that pre-cancer obesity may influence weight gain after cancer diagnosis. Asian women typically have a low prevalence of obesity $[15,17]$. However, little is known about weight change and related factors among Asian women after breast cancer diagnosis. To our knowledge, only one small retrospective study of 260 patients with stage I-III breast cancer has been conducted in a Korean population. This study reported no weight gain after adjuvant treatment [15].

In this report, we describe a comprehensive evaluation of weight change from diagnosis to 18 months after cancer diagnosis and correlates of weight change in a population-based cohort study of 4565 Chinese breast cancer survivors.

\section{Participants and methods}

\section{Study Population and Design}

Study subjects were participants of the Shanghai Breast Cancer Survival Study (SBCSS), a population-based cohort study $[18,19]$. Through the population-based Shanghai Cancer Registry, 6299 women were identified approximately 6 months after diagnosis of incident breast cancer and were invited to participate in the study between April 1, 2002 and December 31, 2006. Of these, 5042 (80.0\%) women provided written, informed consent, enrolled in the study, and completed the baseline survey. Reasons for non-participation included refusals $(12.0 \%)$, moving $(2.7 \%)$, out of town (1.4\%), inability to locate potential participants (1.3\%), and other miscellaneous reasons (2.6\%). The study participants are being followed through in-person interviews administered at approximately 18 months, 36 months, and 60 months after cancer diagnosis. At 18 months post-diagnosis, 4680 cases were contacted. After excluding 108 deceased cases and 11 women who lacked weight data at 18 months post-diagnosis, 4561 participants remained for the current study.

\section{Survey Interviews}

In-person interviews were conducted using structured questionnaires. The following information was collected: cancer diagnosis, cancer-related treatments, relapse/metastasis, disease status, comorbidity, demographics, menstrual and reproductive history, family history of breast cancer, exercise, dietary intake, tea consumption, alcohol consumption, cigarette smoking, complementary and alternative medicine use, and quality of life.

\section{Anthropometric Measurements}

At baseline (approximately 6 months after cancer diagnosis), anthropometric measurements, including height, weight, waist circumference, and hip circumference, were taken by trained interviewers, all of whom were retired medical professionals. All measurements were taken twice according to a standard protocol. Participants were asked to report their weight 1 year before diagnosis, at diagnosis, and at approximately 18 months after diagnosis. Weight at diagnosis was also collected through review of medical charts. Weight changes from 1 year pre-diagnosis to diagnosis and 18 months post-diagnosis were calculated. BMI at diagnosis (weight in kilograms divided by the square of height in meters) was calculated. 


\section{Clinical Factors}

We collected disease- and treatment-related information, including stage of tumor-node metastasis (TNM) at the time of primary diagnosis, estrogen receptor (ER) and progesterone receptor (PR) status, type of surgery, chemotherapy, radiotherapy, immunotherapy, and tamoxifen use. Additionally, medical charts were reviewed to verify diagnosis, treatment, and disease stage information. ER and PR status were included in the analyses in the following joint categories: ER+/PR+ (receptor-positive), ER-/PR- (receptor-negative), and $\mathrm{ER}-/ \mathrm{PR}+$ or ER+/PR- (mixed). The Charlson comorbidity index was created based on a validated comorbidity scoring system [20] and the diagnostic codes from the International Classification of Disease, $9^{\text {th }}$ revision (ICD-9) [21].

\section{Lifestyle Factors}

At the baseline survey, participants were asked to report up to four of the most common exercise activities they participated in between cancer diagnosis and the date of their interview. Information on frequency and duration were obtained for all exercise activities. Each activity was assigned a metabolic equivalent task (MET) score based on the method proposed by Ainsworth et al. [22]. The score for MET-hours per week (MET-hours/week) for each activity was calculated from the hours per week the participant reported engaging in that activity multiplied by the assigned MET score. The values from individual activities were summed to derive a total exercise-MET score.

Habitual dietary intake at baseline was assessed by using an abbreviated 29-item food frequency questionnaire that was designed to capture the intake of meat, fish, cruciferous vegetables, and soy foods. The details of the full-scale dietary assessment questionnaire have been described elsewhere [23]. Information about other lifestyle factors including tea consumption, alcohol consumption, and smoking habits were also obtained through the inperson interview. Information on the amount of tea was collected as weight of dry leaves consumed per month (g/month).

\section{Statistical Analyses}

Mean, standard error (SE), and median of weight change from cancer diagnosis to 18 months after diagnosis were calculated. The t-test or one-way analysis of variance (ANOVA) was applied to compare differences in weight change across sociodemographic, clinical, and lifestyle characteristics. A general linear model (GLM) was used to compute the least square means of weight change with adjustment for age at diagnosis and disease stage. Pearson correlation analysis was performed to examine correlations between selfreported weight and measured weight collected through review of medical charts at the time of cancer diagnosis. For the outcome of 18-month post-diagnosis weight change, women were categorized as having lost weight (lost $>2 \mathrm{~kg}$ ), having maintained weight (weight change $\pm 2 \mathrm{~kg}$, as reference), having moderate weight gain (gained 2-5 kg), or having substantial weight gain (gained $\geq 5 \mathrm{~kg}$ ). Multinomial logistic regression models were conducted to examine associations of weight change status with sociodemographic, clinical, and lifestyle characteristics. Percent (\%) of weight change during the 18-month postdiagnosis period was also calculated: (weight at 18 months post-diagnosis - weight at diagnosis)/(weight at diagnosis) multiplied by 100 . BMI at diagnosis was categorized according to the World Health Organization (WHO) guidelines [24]. The median of 1-year pre-diagnosis weight change was $0 \mathrm{~kg}$ (range: $-25 \sim 14 \mathrm{~kg}$ ), therefore women were divided into 3 three categories based on pre-diagnosis weight change: weight loss $(0.5 \sim 25 \mathrm{~kg})$, weight stable (weight change $=0 \mathrm{~kg}$ ), and weight gain $(1 \sim 14 \mathrm{~kg}$ ). Exercise was categorized by using the median of exercise METs among exercisers as the cut-off points; women who reported no exercise participation served as the reference group. Total dietary intakes of meats, fish, cruciferous vegetables, and soy foods were categorized by quartile distribution. 
Tea consumption was grouped using the median of the consumption of dried tea leaves among current tea drinkers; participants who did not consume tea served as the reference. Variables that were significantly or nearly significantly associated with weight change in univariate analysis were included in the multinomial logistic regression analysis. Disease stage (TNM stage) and ER/PR status were also included in the model.

Tests for trend in the analyses were performed by entering the categorical variables as continuous parameters in the corresponding models. All tests were performed by using Statistical Analysis Software (SAS, version 9.1; SAS Institute, Inc., Cary, North Carolina). The significance levels were set at $\mathrm{P}<0.05$ for two-sided analyses.

\section{Results}

Of the 4561 cases in the study, $61 \%$ gained weight, $27 \%$ lost weight, and $12 \%$ had no weight change between cancer diagnosis and 18 months after diagnosis. Of women who gained weight, $27 \%$ gained $2-5 \mathrm{~kg}$ and $24 \%$ gained $\geq 5 \mathrm{~kg}$. Of women who lost weight, $14 \%$ lost $>2 \mathrm{~kg}$. Regarding the percent of weight change, $37 \%$ of women gained $\geq 5 \%$ of the weight since cancer diagnosis, and $17 \%$ gained $\geq 10 \%$ during the 18 -month post-diagnosis period. At the time of breast cancer diagnosis, $32 \%$ of women were overweight or obese $(\mathrm{BMI} \geq 25)$ and $5 \%$ were obese $(\mathrm{BMI} \geq 30)$, while $36 \%$ were overweight or obese and $6 \%$ were obese at the baseline survey (6 months post-diagnosis). A high correlation coefficient was observed between self-reported weight and weight obtained from medical charts at diagnosis $(r=0.92, \mathrm{P}<0.001)$.

Table 1 presents baseline characteristics of the study participants by weight change. The mean weight change was $1.7 \mathrm{~kg}$ (median: 2; range: -22, 22). Younger women gained more weight than older women. Weight change varied by menopausal status. Premenopausal women gained more weight than postmenopausal women. Women who experienced menopausal symptoms gained more weight than those who did not. Women with a higher comorbidity index gained less weight. Receiving chemotherapy or radiotherapy and having an advanced stage cancer were significantly associated with more weight gain. Pre-diagnosis weight loss or being underweight at diagnosis was significantly related to greater weight gain. Women who had higher dietary intake of meat, fish, and cruciferous vegetables gained more weight than those with lower intakes. Using Chinese medicine and smoking cigarettes were significantly related to more weight gain. There were no differences in weight change by family history of breast cancer, type of surgery, tamoxifen use, or immunotherapy. Similar results were found with adjustment for age at diagnosis and disease stage (data not shown).

Results of multinomial logistic regression analyses for sociodemographic factors are shown in Table 2. Age at diagnosis was inversely related to moderate $\left(\mathrm{P}_{\text {trend }}<0.05\right)$ and substantial weight gain $\left(\mathrm{P}_{\text {trend }}<0.001\right)$; the multivariate adjusted ORs were 1.57 (95\% CI: 0.98-2.51) and 2.14 (95\% CI: 1.30-3.50), respectively, for those with age at diagnosis of less than 40 years as compared with those who were $\geq 60$ years of age at diagnosis and maintained their weight. Premenopausal women had a higher risk for substantial weight gain than postmenopausal women (OR: 1.56; 95\% CI: 1.21-2.00). Education, income, and marital status were not significantly associated with weight change.

Associations between clinical factors and weight change are presented in Table 3. A higher comorbidity index $(\geq 2)$ was significantly associated with more weight loss (OR: 1.42; 95\% CI: $1.08-1.86$ ), and inversely related to moderate weight gain (OR: $0.64 ; 95 \%$ CI: $0.48-0.85$ ) and substantial weight gain (OR: 0.53 ; $95 \%$ CI: $0.38-0.74$ ). Mixed ER/PR status was significantly related to moderate and substantial weight gain as compared with positive ER/ 
PR status; the adjusted ORs were 1.26 (95\% CI: 1.03-1.54) and 1.29 (95\% CI: 1.04-1.60), respectively. Women with TNM stage III-IV breast cancer were more likely to have more substantial weight gain (OR: $1.75 ; 95 \%$ CI: 1.27-2.40) than those with early stage disease (TNM stage 0-I). Relapse/metastasis and cancer-related treatments such as chemotherapy and radiotherapy were not significantly associated with weight change in the multivariate analysis. Tamoxifen use was not associated with weight gain.

Table 4 presents associations of lifestyle factors with weight change. Pre-diagnosis weight gain was marginally associated with weight loss 18 months after diagnosis (OR: 1.26; 95\% CI: 0.99-1.60), while pre-diagnosis weight loss was related to moderate (OR: 1.60 ; 95\% CI: 1.33-1.94) and substantial weight gain (OR: 1.83 ; 95\% CI: 1.50-2.23). Women who were overweight (BMI: 25.0-29.9) and obese (BMI $\geq 30$ ) at diagnosis were more likely to lose weight, the multivariate adjusted ORs were 1.67 (95\% CI: 1.36-2.05) and 2.82 (95\% CI: 2.01-3.96), respectively. Overweight and obese women were less likely to have moderate/ substantial weight gain than those with normal weight. Higher exercise level ( $\geq 8.3$ METhours/week) was marginally related to weight loss (OR: 1.22; 95\% CI: 0.97-1.54). High total dietary intake including meat, fish, cruciferous vegetables, soy foods, and seaweed $\left(\mathrm{P}_{\text {trend }}<0.01\right)$ and cigarette smoking (OR: 2.10; 95\% CI: 1.28-3.45) were related to substantial weight gain. Tea consumption was related to moderate weight gain (OR: 1.43; 95\% CI: 1.03-1.98). No significant association was observed for weight change with alcohol consumption or the use of Chinese medicine.

We also evaluated the percent of weight change after cancer diagnosis and found similar results (data not shown).

\section{Discussion}

In this large, population-based, cohort study, we found that more than three fifths of breast cancer survivors (61\%) gained weight, and the mean weight gain from diagnosis to 18 months after diagnosis was $1.7 \mathrm{~kg}$. Approximately one fourth (24\%) gained $5 \mathrm{~kg}$ or more. We also observed that weight change after breast cancer diagnosis was significantly associated with sociodemographic, clinical, and lifestyle factors. To our knowledge, this is the first population-based cohort study to evaluate weight change and its correlates among Asian women with breast cancer.

Weight gain is a common phenomenon among breast cancer patients and survivors in Western countries $[1,2,4-6,10]$. The Women's Healthy Eating and Living Study (WHEL) observed $2.7 \mathrm{~kg}$ of mean weight gain among 1116 women with stage I-IIIA breast cancer, $60 \%$ of whom reported weight gain, $26 \%$ of whom reported weight loss, and $14 \%$ of whom reported no weight change within 4 years of diagnosis [10]. These statistics are comparable with what we observed in our Chinese population. However, one recent study conducted in Korea found no weight gain after adjuvant treatment among women with stage I-III breast cancer [15]. The mean weight changes in the Korean study were $-0.3 \mathrm{~kg}$ at 1 year and -0.4 $\mathrm{kg}$ at 2 years after cancer treatment, and only $10 \%$ of women gained more than $5 \%$ of baseline body weight at 1 year [15]. The discrepancy between our study and the Korean study may be due to a difference in study design (prospective vs. retrospective), cancer stage (0-IV vs. I-III), study sample size (4565 vs. 260), the timing of the weight change assessment (18 months after diagnosis vs. 12-24 months after cancer treatment), or socioeconomic characteristics.

Several studies have evaluated the association of sociodemographic factors with weight change after breast cancer diagnosis [1, 2, 6, 7, 10-12]. Our findings show that women who were younger at diagnosis gained more weight than older women, which is consistent with 
earlier reports [6, 10]. Similar to Irwin et al. [6], we found no association of weight change with family history of breast cancer or education. The association between menopausal status and weight change remains controversial $[6,7,10,12,15]$. In our study, we found that premenopausal status was significantly related to more weight gain, consistent with some previous studies $[7,12]$ but in disagreement with others $[6,10,15]$. It has been suggested that premenopausal women are more likely to develop amenorrhea or premature menopause over the course of cancer-related treatments and thus are prone to gaining more weight than postmenopausal women [12].

Associations between cancer-related treatments and weight change have been examined with mixed results $[1,6,10,12,15]$. For example, in a cohort of 535 women with newly diagnosed breast cancer, Goodwin et al. observed that adjuvant chemotherapy was a strong and independent clinical predictor of weight gain in Canada [1]. The WHEL study reported similar results [10]. In the Health, Eating, Activity, and Lifestyle (HEAL) study of 514 US women with stage 0-IIIA breast cancer, no association was found between chemotherapy and weight gain, while greater increases in weight were observed among women who were diagnosed with a more advanced disease stage, were younger, were postmenopausal, and decreased their physical activity level between diagnosis to up to 3 years after diagnosis [6]. The Korean study observed that chemotherapy was not associated with weight change after cancer treatment [15], which was similar to the study by Campbell et al. in Canada [12]. Although our univariate analyses and analyses with adjustment for age at diagnosis and disease stage suggested that women who received chemotherapy or radiotherapy gained more weight than their counterparts, such associations disappeared in the multivariate analyses in which sociodemographic and lifestyle factors were further adjusted. This result was consistent with two previous studies $[12,15]$. Although our results suggest that some earlier findings might be due to inadequate adjustment for confounders, there is also the possibility of over-adjustment in our study. In line with the results from the HEAL study [6], we found that women with late-stage breast cancer tended to gain more weight than women with an early stage of the disease. Weight gain after cancer treatment may increase the risk for comorbid conditions such as diabetes and coronary heart disease [25]. The presence of comorbidity at cancer diagnosis was found not to be related with weight change afterward [6]. However, our study indicated that comorbidity immediately after cancer diagnosis was inversely associated with weight gain. Comorbidity may play a different role in weight change among Chinese and Western women with breast cancer.

In our study, one-fourth of women had lost some weight during the year prior to cancer diagnosis. We found that women with pre-diagnosis weight loss had $60-80 \%$ increased risk of moderate to substantial weight gain at 18 months post-diagnosis. We also found that overweight/obese women at diagnosis were more likely to lose weight after cancer diagnosis. Pre-diagnosis weight change and body size at cancer diagnosis may play important roles in the development of weight gain after cancer diagnosis and should be considered when examining the effect of clinical or lifestyle factors on post-diagnosis weight gain.

It has been suggested that modifiable lifestyle factors may be related to weight change in breast cancer patients and survivors [4-6, 10], but the evidence is not consistent. Goodwin et $a l$. found that caloric intake and physical activity did not explain weight gain during the first year after breast cancer diagnosis [1]. In the HEAL study, more weight gain was observed among women who decreased their physical activity between diagnosis and up to 3 years after diagnosis [6]. The WHEL study observed that lower exercise levels and higher energy intake after cancer diagnosis were independently associated with increased risk for weight gain up to 4 years after cancer diagnosis [10]. However, we found no significant association between exercise and weight change between diagnosis and 18 months after diagnosis. In 
our study, $65 \%$ of women reported participating in exercise regularly 6 months after diagnosis, and this rate increased to $74 \%$ at 18 months after diagnosis. It is possible that our non-exercising population may not have been large enough to evaluate the effect of an inactive lifestyle on weight change. On the other hand, the majority of women in our study participated in low to moderate levels of exercise, such as walking and Tai Chi. Among those who participated in regular exercise, the median MET-exercise was 8.3 MET-hours/ week of exercise. Thus, the exercise intensity may not have been high enough to result in weight change. The relatively short follow-up period in our study may be another possible explanation. Further follow-up of our cohort is planned, which would help to understand the long-term effect of exercise participation on weight change among breast cancer survivors.

Our study also showed that higher total dietary intake was significantly related to substantial weight gain. Women who had higher total dietary intake also reported higher meat and vegetable intake in our study. This suggests that high energy intake may increase the risk for weight gain after cancer diagnosis [10]. We also found that cigarette smoking was significantly associated with weight gain, although the estimate was based on a small number of smokers, as few women in this population smoked.

Our study has several strengths. The large, population-based, cohort study design and high response rate minimized selection bias. In-person interviews that collected detailed information on a wide range of sociodemographic, clinical, and lifestyle characteristics allowed a comprehensive evaluation of factors related to weight change after cancer diagnosis. Our study also has some limitations. Weight at 1 year before diagnosis and at diagnosis are based on self-reports. However, previous validation studies have shown that self-reported weight is reasonably accurate [26]. Most previous epidemiological studies have used self-reported weight and weight change or body size after breast cancer diagnosis [9, 27-29]. In our study, self-reported weight at diagnosis was highly correlated with weight at diagnosis obtained from medical charts $(r=0.92)$, indicating that self-reported weight information is quite reliable in our study. The relatively short follow-up period is another limitation. Our ongoing follow-up study will allow us to examine the long-term pattern of weight change and related factors after breast cancer diagnosis.

In conclusion, this large, population-based, cohort study indicates that weight gain during the 18 months after cancer diagnosis is common among breast cancer survivors. Young age at diagnosis, premenopausal status, comorbidity, advanced disease stage, pre-diagnostic weight loss, underweight and obesity, and unhealthy lifestyles were significantly related to weight change. Since weight gain is inversely associated with breast cancer outcomes [8], special attention and advice should be provided to breast cancer patients and survivors who have characteristics associated with weight gain. Lifestyle modification, including adopting a healthy diet and engaging in more exercise, should be encouraged.

\section{Acknowledgments}

The content of this article does not necessarily reflect the position or the policy of the US Government, and no official endorsement should be inferred. The authors thank Dr. Fan Jin for her support in study implementation and the participants and staff members of the SBCSS for making this study possible. The authors also thank Drs. Hui Cai and Wanqing Wen for their assistance in statistical analysis and Ms. Bethanie Hull for her assistance in manuscript preparation.

Funding Sources

This study was supported by a grant from the Department of Defense Breast Cancer Research Program (DAMD 17-02-1-0607 and a grant from the National Institutes of Health, National Cancer Institute (R01 CA118229). 


\section{References}

1. Goodwin PJ, Ennis M, Pritchard KI, McCready D, Koo J, et al. Adjuvant treatment and onset of menopause predict weight gain after breast cancer diagnosis. J Clin Oncol. 1999; 17:120-129. [PubMed: 10458225]

2. Demark-Wahnefried W, Rimer BK, Winer EP. Weight gain in women diagnosed with breast cancer. J Am Diet Assoc. 1997; 97:519-526. [PubMed: 9145091]

3. Chlebowski RT, Aiello E, McTiernan A. Weight loss in breast cancer patient management. J Clin Oncol. 2002; 20:1128-1143. [PubMed: 11844838]

4. Goodwin PJ. Weight gain in early-stage breast cancer: where do we go from here? J Clin Oncol. 2001; 19:2367-2369. [PubMed: 11331314]

5. Demark-Wahnefried W, Peterson BL, Winer EP, Marks L, Aziz N, et al. Changes in weight, body composition, and factors influencing energy balance among premenopausal breast cancer patients receiving adjuvant chemotherapy. J Clin Oncol. 2001; 19:2381-2389. [PubMed: 11331316]

6. Irwin ML, McTiernan A, Baumgartner RN, Baumgartner KB, Bernstein L, et al. Changes in body fat and weight after a breast cancer diagnosis: influence of demographic, prognostic, and lifestyle factors. J Clin Oncol. 2005; 23:774-782. [PubMed: 15681521]

7. Camoriano JK, Loprinzi CL, Ingle JN, Therneau TM, Krook JE, et al. Weight change in women treated with adjuvant therapy or observed following mastectomy for node-positive breast cancer. J Clin Oncol. 1990; 8:1327-1334. [PubMed: 2199619]

8. Kroenke CH, Chen WY, Rosner B, Holmes MD. Weight, weight gain, and survival after breast cancer diagnosis. J Clin Oncol. 2005; 23:1370-1378. [PubMed: 15684320]

9. Loi S, Milne RL, Friedlander ML, McCredie MR, Giles GG, et al. Obesity and outcomes in premenopausal and postmenopausal breast cancer. Cancer Epidemiol Biomarkers Prev. 2005; 14:1686-1691. [PubMed: 16030102]

10. Rock CL, Flatt SW, Newman V, Caan BJ, Haan MN, et al. Factors associated with weight gain in women after diagnosis of breast cancer. Women's Healthy Eating and Living Study Group. J Am Diet Assoc. 1999; 99:1212-1221. [PubMed: 10524383]

11. Freedman RJ, Aziz N, Albanes D, Hartman T, Danforth D, et al. Weight and body composition changes during and after adjuvant chemotherapy in women with breast cancer. J Clin Endocrinol Metab. 2004; 89:2248-2253. [PubMed: 15126549]

12. Campbell KL, Lane K, Martin AD, Gelmon KA, McKenzie DC. Resting energy expenditure and body mass changes in women during adjuvant chemotherapy for breast cancer. Cancer Nurs. 2007; 30:95-100. [PubMed: 17413774]

13. McInnes JA, Knobf MT. Weight gain and quality of life in women treated with adjuvant chemotherapy for early-stage breast cancer. Oncol Nurs Forum. 2001; 28:675-684. [PubMed: 11383182]

14. Harvie MN, Campbell IT, Baildam A, Howell A. Energy balance in early breast cancer patients receiving adjuvant chemotherapy. Breast cancer Res Treat. 2004; 83:201-210. [PubMed: 14758090]

15. Han HS, Lee KW, Kim JH, Kim SW, Kim IA, et al. Weight changes after adjuvant treatment in Korean women with early breast cancer. Breast Cancer Res Treat. 2009; 114:147-153. [PubMed: 18365310]

16. Litton JK, Gonzalez-Angulo AM, Warneke CL, Buzdar AU, Kau SW, et al. Relationship between obesity and pathologic response to neoadjuvant chemotherapy among women with operable breast cancer. J Clin Oncol. 2008; 26:4072-4077. [PubMed: 18757321]

17. World Health Organization, International Association for the Study of Obesity, International Obesity Task Force. The Asia-Pacific Perspective: redefining Obesity and its Treatment. Health Communications Australia Pty Ltd; Melbourne, Australia: 2000.

18. Chen Z, Gu K, Zheng Y, Zheng W, Lu W, et al. The use of complementary and alternative medicine among Chinese women with breast cancer. J Altern Complement Med. 2008; 14:10491055. [PubMed: 18928393] 
19. Lu W, Cui Y, Chen X, Zheng Y, Gu K, et al. Changes in quality of life among breast cancer patients three years post-diagnosis. Breast Cancer Res Treat. 2009; 114:357-369. [PubMed: 18409069]

20. Grunau GL, Sheps S, Goldner EM, Ratner PA. Specific comorbidity risk adjustment was a better predictor of 5-year acute myocardial infarction mortality than general methods. J Clin Epidemiol. 2006; 59:274-280. [PubMed: 16488358]

21. Department of Health and Human Services. The international classification of diseases. 9th rev. ed. Clinical modification, ICD-9-CM. U.S Government Printing Office; Washington, DC: 1998.

22. Ainsworth BE, Haskell WL, Leon AS, Jacobs DR Jr, Montoye HJ, et al. Compendium of physical activities: classification of energy costs of human physical activities. Med Sci Sports Exerc. 1993; 25:71-80. [PubMed: 8292105]

23. Boyapati SM, Shu XO, Ruan ZX, Dai Q, Cai Q, et al. Soyfood intake and breast cancer survival: a followup of the Shanghai Breast Cancer Study. Breast Cancer Res Treat. 2005; 92:11-17. [PubMed: 15980986]

24. World Health Organization. Obesity: Preventing and managing the global epidemic. Report of a WHO consultation of obesity. Geneva: 1997. p. 3-5.

25. Rock CL, Demark-Wahnefried W. Nutrition and survival after the diagnosis of breast cancer: a review of the evidence. J Clin Oncol. 2002; 20:3302-3316. [PubMed: 12149305]

26. Spencer EA, Appleby PN, Davey GK, Key TJ. Validity of self-reported height and weight in 4808 EPIC-Oxford participants. Public Health Nutr. 2002; 5:561-565. [PubMed: 12186665]

27. Daling JR, Malone KE, Doody DR, Johnson LG, Gralow JR, et al. Relation of body mass index to tumor markers and survival among young women with invasive ductal breast carcinoma. Cancer. 2001; 92:720-729. [PubMed: 11550140]

28. Eng SM, Gammon MD, Terry MB, Kushi LH, Teitelbaum SL, et al. Body size changes in relation to postmenopausal breast cancer among women on Long Island, New York. Am J Epidemiol. 2005; 162:229-237. [PubMed: 15987723]

29. Whiteman MK, Hillis SD, Curtis KM, McDonald JA, Wingo PA, et al. Body mass and mortality after breast cancer diagnosis. Cancer Epidemiol Biomarkers Prev. 2005; 14:2009-2014. [PubMed: 16103453] 


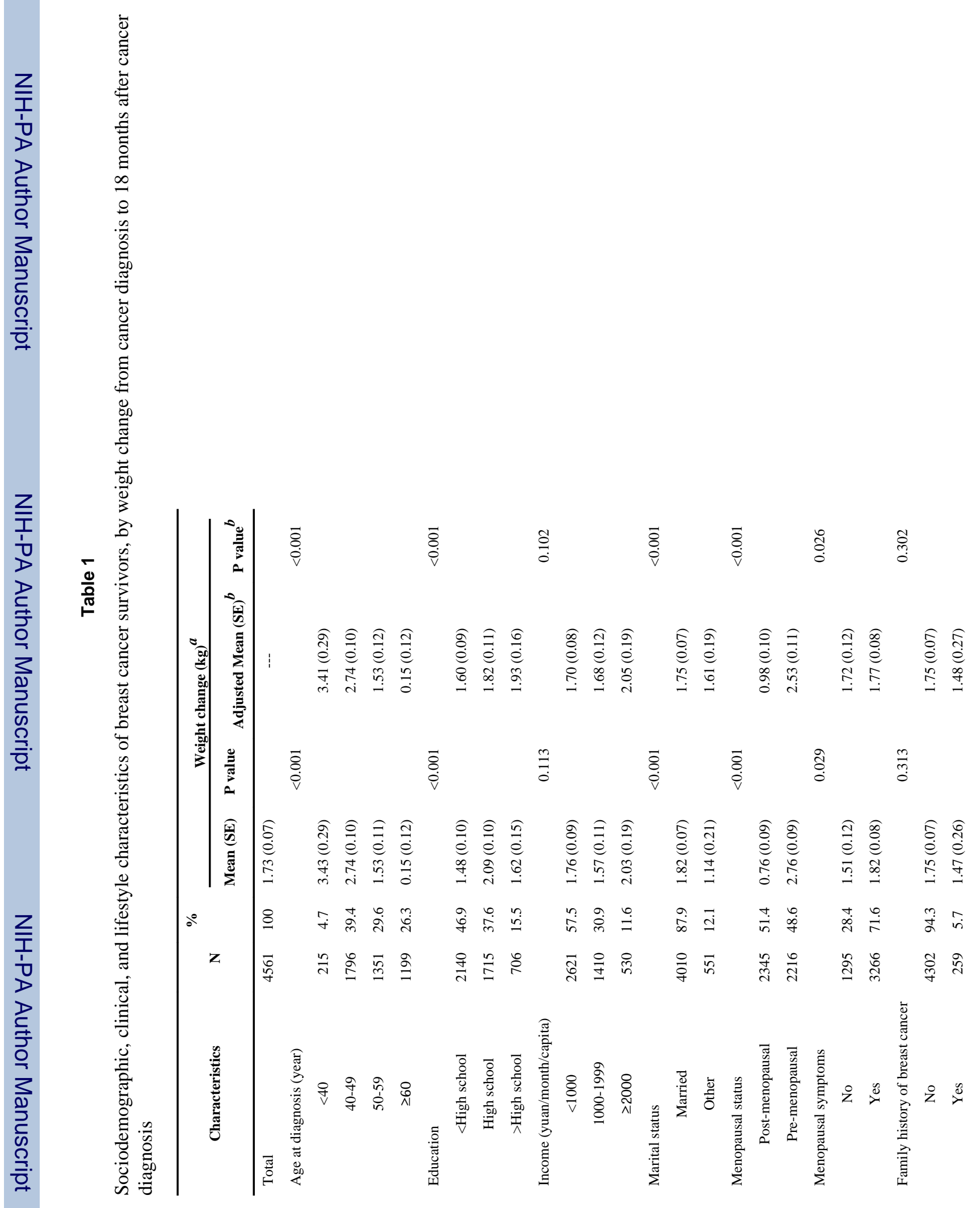




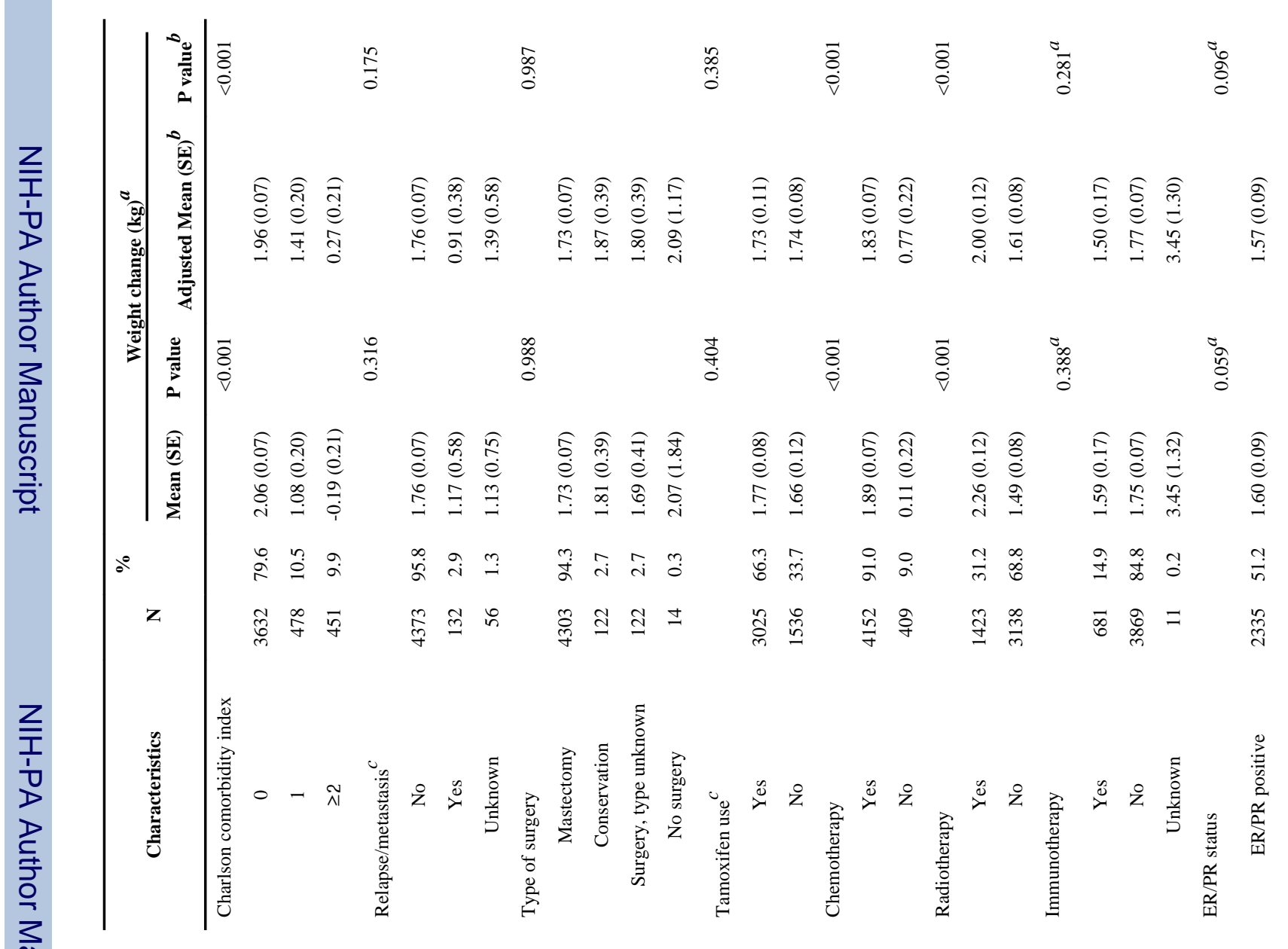




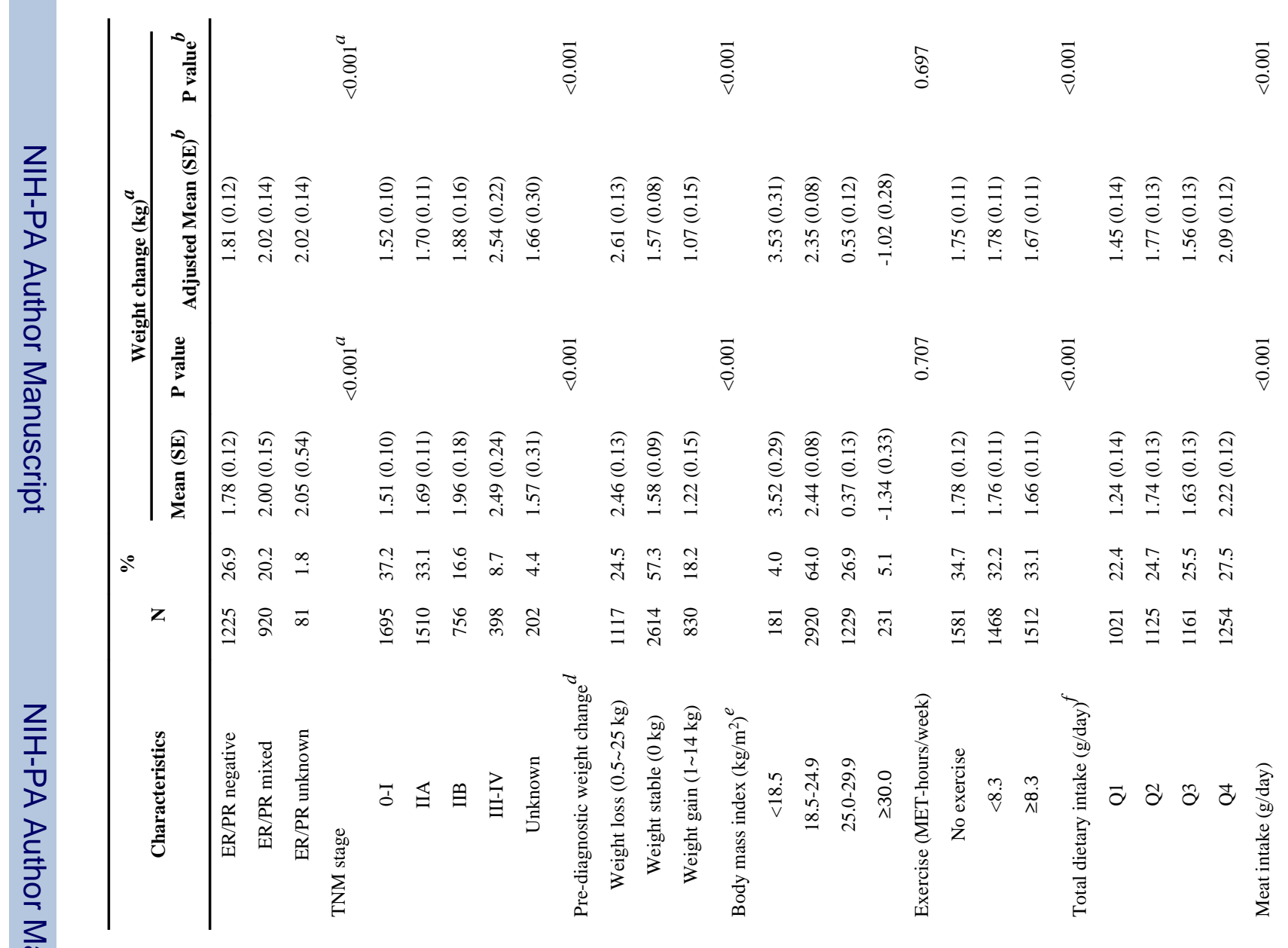




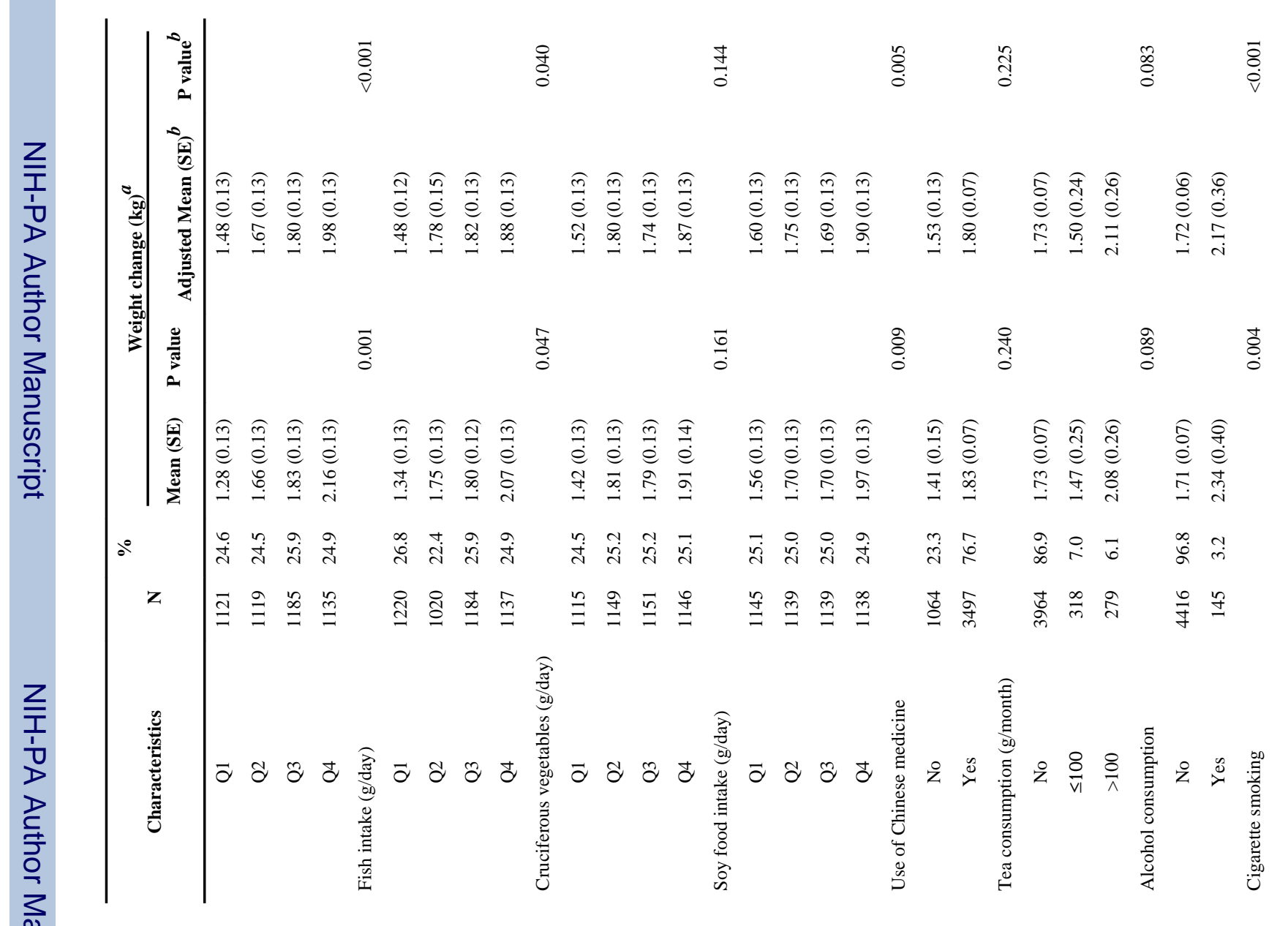




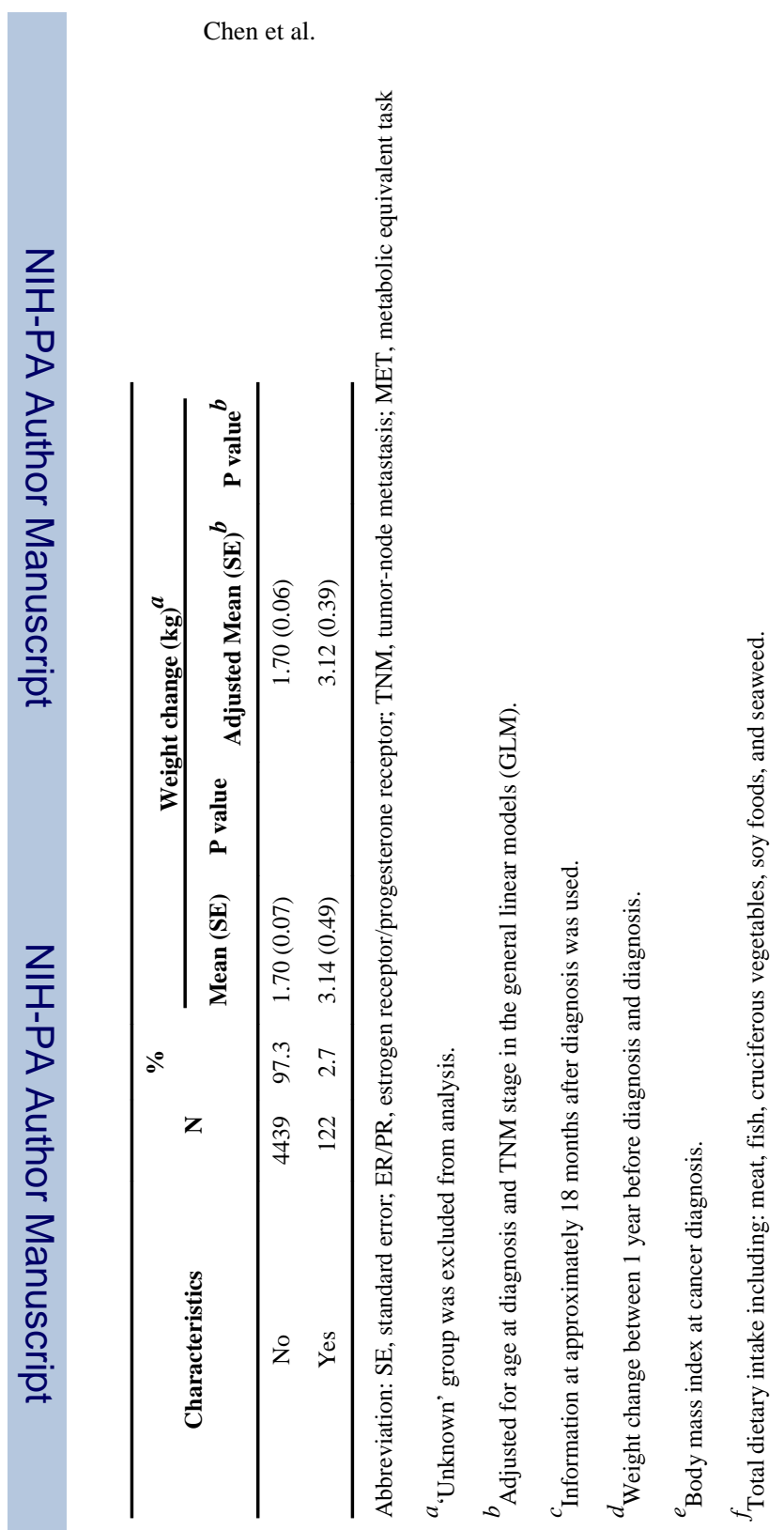

Page 14

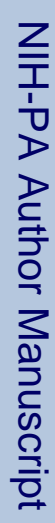

Nutr Cancer. Author manuscript; available in PMC 2012 May 1. 
Table 2

Multinomial logistic regression models for sociodemographic factors related to weight change from diagnosis to 18 months after diagnosis among breast cancer survivors ${ }^{a}$

\begin{tabular}{|c|c|c|c|}
\hline Characteristics & Weight loss $<2 \mathrm{~kg}$ OR $(95 \% \mathrm{CI})$ & Weight gain 2-5kg OR (95\% CI) & Weight gain $\geq 5 \mathrm{~kg}$ OR $(95 \% \mathrm{CI})$ \\
\hline \multicolumn{4}{|l|}{ Age at diagnosis (year) } \\
\hline$\geq 60$ & 1.00 & 1.00 & 1.00 \\
\hline $50-59$ & $0.88(0.68-1.14)$ & $1.35(1.08-1.71)$ & $1.60(1.22-2.10)$ \\
\hline $40-49$ & $0.85(0.58-1.24)$ & $1.31(0.97-1.78)$ & $2.05(1.46-2.87)$ \\
\hline$<40$ & $0.79(0.39-1.59)$ & $1.57(0.98-2.51)$ & $2.14(1.30-3.50)$ \\
\hline$P$ value for trend & 0.286 & 0.039 & $<0.001$ \\
\hline \multicolumn{4}{|l|}{ Education } \\
\hline$<$ High school & 1.00 & 1.00 & 1.00 \\
\hline High school & $1.07(0.86-1.33)$ & $1.04(0.87-1.24)$ & $0.94(0.78-1.14)$ \\
\hline$>$ High school & $0.93(0.68-1.26)$ & $0.90(0.70-1.16)$ & $0.79(0.60-1.04)$ \\
\hline$P$ value for trend & 0.858 & 0.651 & 0.121 \\
\hline \multicolumn{4}{|c|}{ Income (yuan/month/capita) } \\
\hline$<1000$ & 1.00 & 1.00 & 1.00 \\
\hline $1000-1999$ & $0.94(0.76-1.18)$ & $0.93(0.78-1.11)$ & $0.82(0.68-1.00)$ \\
\hline$\geq 2000$ & $1.00(0.70-1.42)$ & $1.07(0.81-1.40)$ & $0.99(0.74-1.32)$ \\
\hline$P$ value for trend & 0.784 & 0.959 & 0.319 \\
\hline \multicolumn{4}{|l|}{ Marital status } \\
\hline Married & 1.00 & 1.00 & 1.00 \\
\hline Other & $1.01(0.77-1.33)$ & $0.87(0.68-1.11)$ & $0.95(0.73-1.24)$ \\
\hline \multicolumn{4}{|l|}{ Menopausal status } \\
\hline Post-menopausal & 1.00 & 1.00 & 1.00 \\
\hline Pre-menopausal & $0.90(0.66-1.23)$ & $1.19(0.94-1.50)$ & $1.56(1.21-2.00)$ \\
\hline
\end{tabular}

Abbreviations: OR, odds ratio; 95\% CI, 95\% confidence interval.

${ }^{a}$ Women who had maintained weight (weight change $\pm 2 \mathrm{~kg}$ ) during the 18-month post-diagnosis period were treated as the reference. Adjusted for menopausal symptoms, comorbidity, chemotherapy, radiotherapy, estrogen receptor/progesterone receptor status, tumor-node metastasis stage, prediagnosis weight change, body mass index at diagnosis, exercise, dietary intake, use of Chinese medicine, tea consumption, alcohol consumption, cigarette smoking, and other variables in this table. 
Table 3

Multinomial logistic regression models for clinical factors related to weight change from diagnosis to 18 months after diagnosis among breast cancer survivors ${ }^{a}$

\begin{tabular}{|c|c|c|c|}
\hline Characteristics & Weight loss $<2 \mathrm{~kg}$ OR $(95 \% \mathrm{CI})$ & Weight gain 2-5kg OR $(95 \% \mathrm{CI})$ & Weight gain $\geq 5 \mathrm{~kg}$ OR $(95 \% \mathrm{CI})$ \\
\hline \multicolumn{4}{|l|}{ Menopausal symptoms } \\
\hline No & 1.00 & 1.00 & 1.00 \\
\hline Yes & $1.20(0.97-1.49)$ & $1.01(0.84-1.19)$ & $0.90(0.75-1.08)$ \\
\hline \multicolumn{4}{|c|}{ Charlson comorbidity index } \\
\hline 0 & 1.00 & 1.00 & 1.00 \\
\hline 1 & $0.89(0.66-1.20)$ & $1.03(0.80-1.32)$ & $0.78(0.58-1.05)$ \\
\hline$\geq 2$ & $1.42(1.08-1.86)$ & $0.64(0.48-0.85)$ & $0.53(0.38-0.74)$ \\
\hline$P$ value for trend & 0.034 & 0.010 & $<0.001$ \\
\hline \multicolumn{4}{|l|}{ Chemotherapy } \\
\hline No & 1.00 & 1.00 & 1.00 \\
\hline Yes & $0.94(0.69-1.28)$ & $1.06(0.80-1.42)$ & $1.34(0.94-1.91)$ \\
\hline \multicolumn{4}{|l|}{ Radiotherapy } \\
\hline No & 1.00 & 1.00 & 1.00 \\
\hline Yes & $1.06(0.84-1.34)$ & $1.17(0.97-1.40)$ & $1.15(0.95-1.39)$ \\
\hline \multicolumn{4}{|l|}{ ER/PR status } \\
\hline ER/PR positive & 1.00 & 1.00 & 1.00 \\
\hline ER/PR negative & $0.92(0.73-1.16)$ & $1.00(0.83-1.19)$ & $1.09(0.90-1.33)$ \\
\hline ER/PR mixed & $1.10(0.86-1.41)$ & $1.26(1.03-1.54)$ & $1.29(1.04-1.60)$ \\
\hline ER/PR unknown & $0.55(0.25-1.19)$ & $0.91(0.50-1.66)$ & $1.24(0.68-2.25)$ \\
\hline \multicolumn{4}{|l|}{ TNM stage } \\
\hline 0-I & 1.00 & 1.00 & 1.00 \\
\hline IIA & $0.97(0.77-1.21)$ & $1.03(0.86-1.24)$ & $1.09(0.90-1.33)$ \\
\hline IIB & $0.91(0.68-1.22)$ & $0.99(0.78-1.26)$ & $1.30(1.01-1.66)$ \\
\hline III-IV & $1.09(0.75-1.59)$ & $1.14(0.83-1.56)$ & $1.75(1.27-2.40)$ \\
\hline Unknown & $1.33(0.84-2.11)$ & $0.99(0.66-1.49)$ & $1.52(1.01-2.27)$ \\
\hline
\end{tabular}

Abbreviations: OR, odds ratio; 95\% CI, 95\% confidence interval; ER/PR, estrogen receptor/progesterone receptor; TNM, tumor-node metastasis.

${ }^{a}$ Women who had maintained weight (weight change $\pm 2 \mathrm{~kg}$ ) during the 18-month post-diagnosis period were treated as the reference. Adjusted for age at diagnosis, education, income, marital status, menopausal status, pre-diagnosis weight change, body mass index at diagnosis, exercise, dietary intake, use of Chinese medicine, tea consumption, alcohol consumption, cigarette smoking, and other variables in this table. 
Table 4

Multinomial logistic regression models for lifestyle factors related to weight change from diagnosis to 18 months after diagnosis among breast cancer survivors ${ }^{a}$

\begin{tabular}{|c|c|c|c|}
\hline Characteristics & Weight loss $<2 \mathrm{~kg}$ OR $(95 \% \mathrm{CI})$ & Weight gain 2-5kg OR $(95 \% \mathrm{CI})$ & Weight gain $\geq 5 \mathrm{~kg}$ OR $(95 \% \mathrm{CI})$ \\
\hline \multicolumn{4}{|l|}{ Pre-diagnosis weight change } \\
\hline Weight loss $(0.5 \sim 25 \mathrm{~kg})$ & $1.03(0.80-1.31)$ & $1.60(1.33-1.94)$ & $1.83(1.50-2.23)$ \\
\hline Weight stable $(0 \mathrm{~kg})$ & 1.00 & 1.00 & 1.00 \\
\hline Weight gain (1-14 kg) & $1.26(0.99-1.60)$ & $0.96(0.78-1.18)$ & $0.82(0.66-1.03)$ \\
\hline \multicolumn{4}{|l|}{ Body mass index $\left(\mathrm{kg} / \mathrm{m}^{2}\right)$} \\
\hline$<18.5$ & $0.38(0.15-0.95)$ & $1.45(0.98-2.14)$ & $1.35(0.90-2.03)$ \\
\hline $18.5-24.9$ & 1.00 & 1.00 & 1.00 \\
\hline $25.0-29.9$ & $1.67(1.36-2.05)$ & $0.74(0.62-0.89)$ & $0.52(0.42-0.64)$ \\
\hline$\geq 30.0$ & $2.82(2.01-3.96)$ & $0.48(0.31-0.74)$ & $0.41(0.25-0.67)$ \\
\hline \multicolumn{4}{|l|}{ Exercise (MET-hours/week) } \\
\hline No exercise & 1.00 & 1.00 & 1.00 \\
\hline$<8.3$ & $0.93(0.73-1.17)$ & $0.99(0.82-1.19)$ & $0.96(0.78-1.16)$ \\
\hline$\geq 8.3$ & $1.22(0.97-1.54)$ & $0.99(0.82-1.19)$ & $0.94(0.77-1.15)$ \\
\hline$P$ vaule for trend & 0.090 & 0.911 & 0.548 \\
\hline \multicolumn{4}{|l|}{ Total dietary intake (g/day) $b$} \\
\hline Q1 & 1.00 & 1.00 & 1.00 \\
\hline Q2 & $0.85(0.65-1.12)$ & $0.88(0.70-1.10)$ & $1.03(0.81-1.31)$ \\
\hline Q3 & $1.13(0.87-1.48)$ & $1.18(0.94-1.47)$ & $1.18(0.93-1.51)$ \\
\hline Q4 & $0.98(0.74-1.29)$ & $1.13(0.90-1.41)$ & $1.43(1.12-1.82)$ \\
\hline$P$ vaule for trend & 0.590 & 0.056 & 0.001 \\
\hline \multicolumn{4}{|l|}{ Use of Chinese medicine } \\
\hline No & 1.00 & 1.00 & 1.00 \\
\hline Yes & $0.83(0.66-1.02)$ & $1.00(0.83-1.21)$ & $0.94(0.77-1.14)$ \\
\hline \multicolumn{4}{|l|}{ Tea consumption (g/month) } \\
\hline No & 1.00 & 1.00 & 1.00 \\
\hline$\leq 100$ & $1.05(0.73-1.51)$ & $0.98(0.73-1.32)$ & $0.89(0.65-1.24)$ \\
\hline$>100$ & $1.07(0.70-1.62)$ & $1.43(1.03-1.98)$ & $1.38(0.98-1.96)$ \\
\hline$P$ vaule for trend & 0.727 & 0.069 & 0.183 \\
\hline \multicolumn{4}{|l|}{ Alcohol consumption } \\
\hline No & 1.00 & 1.00 & 1.00 \\
\hline Yes & $0.98(0.55-1.77)$ & $0.95(0.60-1.50)$ & $1.17(0.75-1.84)$ \\
\hline \multicolumn{4}{|l|}{ Cigarette smoking } \\
\hline No & 1.00 & 1.00 & 1.00 \\
\hline Yes & $1.03(0.55-1.94)$ & $1.16(0.68-1.98)$ & $2.10(1.28-3.45)$ \\
\hline
\end{tabular}

Abbreviation: OR, odds ratio; 95\% CI, 95\% confidence interval; MET, metabolic equivalent task.

${ }^{a}$ Women who had maintained weight (weight change $\pm 2 \mathrm{~kg}$ ) during the 18-month post-diagnosis period were treated as the reference. Adjusted for age at diagnosis, education, income, marital status, menopausal status, menopausal symptoms, comorbidity, chemotherapy, radiotherapy, ER/PR status, tumor-node metastasis stage, and other variables in this table. 
${ }^{b}$ Total dietary intake including: meat, fish, cruciferous vegetables, soy foods, and seaweed. 